\title{
Solving the 0/1 Knapsack Problem by a Biomolecular DNA Computer
}

\author{
Hassan Taghipour, ${ }^{1}$ Mahdi Rezaei, ${ }^{2}$ and Heydar Ali Esmaili ${ }^{1}$ \\ ${ }^{1}$ Department of Pathology, Tabriz University of Medical Sciences, Tabriz, Iran \\ ${ }^{2}$ Department of Theoretical Physics and Astrophysics, University of Tabriz, Tabriz 51664, Iran \\ Correspondence should be addressed to Hassan Taghipour; taghipourh@yahoo.com
}

Received 2 November 2012; Revised 11 January 2013; Accepted 11 January 2013

Academic Editor: Bhaskar Dasgupta

Copyright (c) 2013 Hassan Taghipour et al. This is an open access article distributed under the Creative Commons Attribution License, which permits unrestricted use, distribution, and reproduction in any medium, provided the original work is properly cited.

Solving some mathematical problems such as NP-complete problems by conventional silicon-based computers is problematic and takes so long time. DNA computing is an alternative method of computing which uses DNA molecules for computing purposes. DNA computers have massive degrees of parallel processing capability. The massive parallel processing characteristic of DNA computers is of particular interest in solving NP-complete and hard combinatorial problems. NP-complete problems such as knapsack problem and other hard combinatorial problems can be easily solved by DNA computers in a very short period of time comparing to conventional silicon-based computers. Sticker-based DNA computing is one of the methods of DNA computing. In this paper, the sticker based DNA computing was used for solving the $0 / 1$ knapsack problem. At first, a biomolecular solution space was constructed by using appropriate DNA memory complexes. Then, by the application of a sticker-based parallel algorithm using biological operations, knapsack problem was resolved in polynomial time.

\section{Introduction}

DNA encodes the genetic information of cellular organisms. The unique and specific structure of DNA makes it one of the favorite candidates for computing purposes. In comparison with conventional silicon-based computers, DNA computers have massive degrees of miniaturization and parallelism. By recent technology, about $10^{18}$ DNA molecules can be produced and placed in a medium-sized laboratory test tube. Each of these DNA molecules could act as a small processor. Biological operations such as hybridization, separation, setting, and clearing can be performed simultaneously on all of these DNA strands. Thus, in an in vitro assay, we could handle about $10^{18}$ DNA molecules or we can say that $10^{18}$ data processors can be executed in parallel.

In 1994, Adleman introduced the DNA computing as a new method of parallel computing [1]. Adleman succeeded in solving seven-point Hamiltonian path problem solely by manipulating DNA molecules and suggested that DNA could be used to solve complex mathematical problems.
In 1999, a new model of DNA computing (sticker model) was introduced by Roweis et al. [2]. This model has a kind of random access memory that requires no strand extension, uses no enzymes, and its materials are reusable. Stickerbased DNA computing has potential capability for being a universal method in DNA computing. Roweis et al. [2] also proposed specific machine architecture for implementing the sticker model as a microprocessor-controlled parallel robotic workstation. Thus, the operations used in sticker model can be performed on fully automated devices, which is helpful in reducing the error rates of operations.

In this paper, we applied sticker model for solving the knapsack problem which is one of the NP-complete problems.

The paper is organized as follows. Section 2 introduces the DNA structure and various DNA computing models and discusses about the sticker based DNA computing and biological operations which are used in sticker model. Section 3 introduces a DNA-based algorithm for solving the knapsack problem in sticker model. 


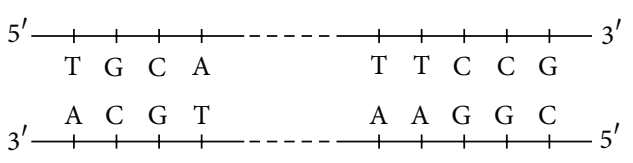

FIgURE 1: A DNA molecule.

\section{Basics of DNA Computing}

2.1. Structure of DNA and DNA Computing Models. DNA is a polymeric and a double-stranded molecule which is composed of monomers called nucleotides. Nucleotides are building blocks of DNA, and each of them contains three components: sugar, phosphate group, and nitrogenous base. There are four different nitrogenous bases which contribute in DNA structure: Thymine (T) and Cytosine (C) which are called pyrimidines and Adenine (A) and Guanine (G) which are called purines. Because nitrogenous bases are variable components of nucleotides, different neucleotides are distinguished by nitrogenous bases which contribute in their structure. For this reason, the name of the bases are used to refer to the neucleotides, and the neucleotides are simply represented as A, G, C, and T. The nucleotides are linked together by phosphodiester bonds and form a single-stranded DNA (ssDNA). A ssDNA molecule can be likened to a string consisting of a combination of four different symbols, A, G, $\mathrm{C}$, and T. Mathematically, this means that we have a fourletter alphabet $\sum=\{A, G, C, T\}$ to encode information. Two ssDNA molecules join together to form a double-stranded DNA (DsDNA) based on complementary rule: "A" always pairs with "T," and likewise " $C$ " pairs with "G." In Figure 1, a schematic picture of DNA is shown.

DNA computing was initially developed by Adleman in 1994. Adleman resolved an instance of Hamiltonian path problem just by handling the DNA molecules [1]. In 1995, Lipton presented a method for solving the satisfiability (SAT) problem [3]. Adleman-Lipton model can be used to solve different NP-complete problems. In Adleman-Lipton model, DNA splints are used for the construction of solution space. Adleman $[4,5]$ also presented a molecular algorithm for solving the 3-coloring problem. Chang and Guo [6-8] showed that the DNA operations in Adelman-Lipton model could be used for developing DNA algorithms to resolve the dominating set problem, the vertex cover problem, the maximal clique problem, and the independent set problem.

In 1999, Roweis et al. [2] introduced the Sticker based DNA computing model and applied it in solving the minimal set cover problem, and this model also was applied for breaking the Data Encryption Standard (DES) [9]. In our previous work, we also applied sticker based model for solving the independent set problem [10].

Other than Adleman-Lipton and Sticker based models, other various models are also proposed in DNA computing by researchers. Quyang et al. [11] solved the maximal clique problem using DNA molecules and restriction endonuclease enzymes. Amos et al. [12, 13] described a DNA computation model using restriction endonuclease enzymes instead of successive cycles of separation by DNA hybridization, which can reduce the error rate of computation. Hagiya et al. [14] proposed a new method of DNA computing that involves a self-acting DNA molecule containing both the input, program, and working memory. In this method, a singlestranded DNA molecule consists of an input segment on the $5^{\prime}$ end, followed by a formula (program) segment, followed by a spacer, and finally with a "head" on the 3 ' end that moves and performs the computation. Another method for DNA computation is "computation by self-assembly." Winfree et al. [15-17] introduced a linear and 2-dimensional self-assembly model.

The surface-based model was introduced by Liu et al. [18]. This model uses DNA molecules attached to a solid surface, instead of DNA molecules floating in a solution. The surfacebased model was used by Taghipour et al. for solving the dominating set problem [19]. The computing by blocking was introduced by Rozenberg and Spaink [20]. This model uses a novel approach to filter the DNA molecules. Instead of separating the DNA strands to distinct tubes, or destroying and removing the DNA molecules that do not contribute to finding a solution, it blocks (inactivates) them in a way that the blocked strands can be considered as nonexistent during the subsequent steps of computation.

2.2. Sticker-Based DNA Computation. The sticker model was introduced by Roweis et al. [2]. In this model, there is a memory strand with $N$ bases in length subdivided into $K$ nonoverlapping regions each $M$ bases long $(N \geq M K)$. $M$ can be, for example, 20. The substrands (bit regions) are significantly different from each other. One sticker is designed for each subregion; each sticker has $M$ bases long and is complementary to one and only one of the $K$ memory regions. If a sticker is annealed to its corresponding region on memory strand, then the particular region is said to be on. If no sticker is annealed to a region, then the corresponding bit is off. Each memory strand along with its annealed stickers is called memory complex. In sticker model, a tube is a collection of memory complexes, composed of large number of identical memory strands each of which has stickers annealed only at the required bit positions. This method of representation of information differs from other methods in which the presence or absence of a particular subsequence in a strand corresponded to a particular bit being on or off. In sticker model, each possible bit string is represented by a unique association of memory strands and stickers. This model has a kind of random access memory that requires no strand extension and uses no enzymes [2]. Indeed, in the sticker model, memory strands are used as registers, and stickers are used to write and erase information in the registers.

Another conception in sticker model is $(K, L)$ library. Each $(K, L)$ library contains memory complexes with $K$ bit regions, the first $L$ bit regions are either on or off, in all possible ways, whereas the remaining $K-L$ bit regions are off. The last $K-L$ bit regions can be used for intermediate data storage. In every $(K, L)$ library, there are at least $2^{L}$ memory complexes. In Figure 2, a memory complex with 7 bit regions representing the binary number 1100101 is shown. 
FIGURE 2: A memory complex representing 1100101.

2.3. Biological Operations in Sticker Model. There are four principal operations in sticker model: combination, separation, setting, and clearing [2]. We also defined a new operation called "divide" which is used in the construction of solution space [10]. Here, we briefly discuss about these operations.

(1) Combine $\left(T_{0}, T_{1}\right.$, and $\left.T_{2}\right)$. The memory complexes from the tubes $T_{1}$ and $T_{2}$ are combined to form a new tube, $T_{0}$, simply the contents of $T_{1}$ and $T_{2}$ are poured into the tube $T_{0}$. $\left(T_{0}=T_{1} \cup T_{2}\right)$.

(2) Separate $\left(T_{0}, i\right) \rightarrow\left(T^{+}, T^{-}\right)$. This operation creates two new tubes $T^{+}$and $T^{-} ; T^{+}$contains the memory complexes having the $i$ th bit on $\left(T^{+}=+\left(T_{0}, i\right)\right)$, and $T^{-}$contains the memory complexes having the $i$ th bit off $\left(T^{-}=-\left(T_{0}, i\right)\right)$.

(3) Set $\left(T_{0}, i\right)$. The $i$ th bit region on every memory complex in tube $T_{0}$ is set to 1 or turned on.

(4) Clear $\left(T_{0}, i\right)$. The $i$ th bit region on every memory complex in tube $T_{0}$ is set to 0 or turned off.

(5) Divide $\left(T_{0}, T_{1}\right.$, and $\left.T_{2}\right)$. By this operation, the contents of tube $T_{0}$ is divided into two equal portions and poured into the tubes $T_{1}$ and $T_{2}$.

\section{Solving the 0/1 Knapsack Problem in Sticker-Based DNA Computers}

3.1. Definition of the Knapsack Problem. Knapsack problem is one of the classical optimization problems which have two variants: the $0 / 1$ and fractional knapsack problems.

The $0 / 1$ knapsack problem is posed as follows.

There are $n$ items $I_{1}, I_{2}, I_{3}, \ldots, I_{n}$; each item $I_{j}$ has a weight $W_{j}$ and a value $V_{j}$, where $W_{j}$ and $V_{j}$ are integers. We have a knapsack which its capacity (weight) is $C$, where $C$ is also an integer. We want to take the most valuable set of items that fit in our knapsack. Which items should we take? This is called the $0 / 1$ or binary knapsack problem because each item must either be taken or left behind; we cannot take a fractional amount of an item.

In the fractional knapsack problem, the setup is the same, but we can take fractions of items, rather than having to make a binary (0-1) choice for each item. The fractional knapsack problem is solvable by a greedy strategy, where as the $0 / 1$ knapsack problem is not. The $0 / 1$ knapsack problem has been proved to be an NP-complete problem [21].

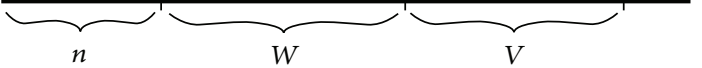

Figure 3: Memory strand with at least $n+W+V$ bit regions.

\subsection{Construction of Sticker Based DNA Solution Space for Knapsack Problem}

3.2.1. Designing Appropriate DNA Memory Complexes. As discussed before, there are $n$ items $I_{1}, I_{2}, I_{3}, \ldots, I_{n}$; each item $I_{j}$ has a weight $W_{j}$ and a value $V_{j}$, where $W_{j}$ and $V_{j}$ are integers. Let us consider that the total weight of items is $W$ and total value of items is $V$.

$$
\begin{gathered}
W=W_{1}+W_{2}+W_{3}+\cdots+W_{n}=\sum_{j=1}^{n} W_{j}, \\
V=V_{1}+V_{2}+V_{3}+\cdots+V_{n}=\sum_{j=1}^{n} V_{j}
\end{gathered}
$$

$n=$ total number of items.

We start with $2^{n}$ or more identical memory strands, which each of them has at least $n+W+V$ bit regions. (Figure 3 ) The first $n$ bit regions (bit regions 1 to $n$ ) are used to represent $n$ items, the middle $W$ bit regions (bit regions $n+1$ to $n+$ $W$ ) represent the total weight of items $W$, and the next $V$ bit regions (bit regions $n+W+1$ to $n+W+V$ ) represent the total value of items $V$. Each bit region, for example can have 20 neucleotides, furthermore, every memory strand at least contains $20(n+W+V)$ neucleotides.

3.2.2. Production of DNA Memory Complexes Which Represent All Possible Subsets of Items. It is clear that a set of $n$ items has $2^{n}$ subsets and each of these subsets has its own weight and value. For construction of solution space, it is essential to represent all subsets of items by appropriate DNA memory complexes. Furthermore, by using at least $2^{n}$ or more memory strands and making the first $n$ bit regions on or off in all possible ways, we represent all $2^{n}$ subsets of items by DNA memory complexes. On the other hand, simply we design a $(n+W+V, n)$ library. For this purpose, (Procedure 1$)$ is proposed.

Procedure 1 has $n$ divide, $n$ set and $n$ combine operations. At the end of procedure, tube $T_{0}$ contains all of the memory complexes which each of them represent one of the subsets of items.

3.2.3. Representing the Weight and Value of Each Subset on DNA Memory Complexes. In this step, based on the items which are present in subsets, and by annealing corresponding stickers in $W$ and $V$ regions of memory strands, the total weight and value of subsets are represent on memory complexes. Note, each item $I_{j}$ has a weight $W_{j}$ and a value $V_{j}$, thus, 
(1) Input $\left(T_{0}\right)$, where $T_{0}$ contains $2^{n}$ or more memory strands with at least $(n+W+V)$ bit regions.

(2) For $i=1$ to $n$, where $n$ is the total number of items

(a) Divide $\left(T_{0}, T_{1}, T_{2}\right)$

(b) Set $\left(T_{1}, i\right)$

End for

(c) Combine $\left(T_{0}, T_{1}, T_{2}\right)$

Procedure 1

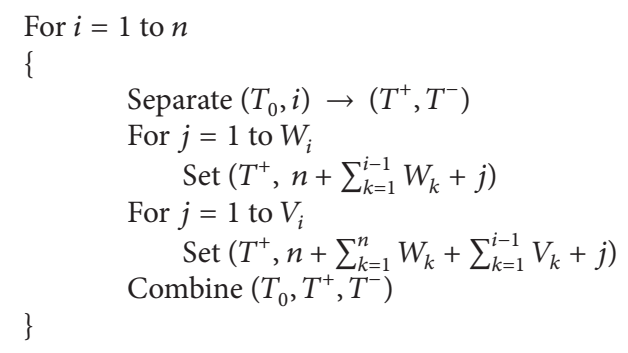

Procedure 2

for each item $I_{j}, W_{j}$ numbers of stickers are annealed to $W$ region and $V_{j}$ numbers of stickers are annealed to $V$ region on memory strands. Furthermore, the numbers of annealed stickers in $W$ and $V$ regions represent the weight and value of corresponding subset, respectively. Procedure 2 is proposed for representing the weight and value of each subset.

Now, our solution space is completely produced and contains at least $2^{n}$ memory complexes, which each of them represent one of the subsets of items, and the numbers of annealed stickers in $W$ and $V$ regions represent the weight and value of corresponding subset, respectively.

3.3. DNA Algorithm for Solving the 0/1 Knapsack Problem. Algorithm 1 is proposed for solving the 0/1 knapsack problem.

According to the steps in the algorithm, the knapsack problem can be resolved by sticker based DNA computation in polynomial time.

By the execution of step 1, the memory complexes without any annealed stickers in $W$ region (represent the subset $\emptyset$ ) are placed in tube $T_{0}$, the memory complexes with only one annealed sticker (represent the subsets of items which their weight are 1) are placed in tube $T_{1}$, the memory complexes with 2 annealed stickers (represent the subsets of items which their weight are 2) are placed in tubes $T_{2}$, the memory complexes with 3 annealed stickers (represent the subsets of items which their weight are 3) are placed in tube $T_{3}$, and finally, the tube $T_{W}$ contains the memory complexes witch all bit regions located in $W$ region are turned to "on" (represent the subset which contains all items). On the other hands, step 1 is a sorting procedure and sorts memory complexes according to the number of annealed stickers in $W$ region. In this step, $W+1$ tubes are produced $\left(T_{0}, T_{1}, T_{2}, \ldots, T_{W}\right)$, and number of every tube indicate the number of annealed stickers in $W$ region. Step 1 contains $W(W+1) / 2$ separate and $W(W+1) / 2$ combine operations, or totally it contains $W(W+1)$ operations.

In step 2 of algorithm, the contents of tubes $T_{c+1}, T_{c+2}, T_{c+3}, \ldots, T_{W}$ are discarded, because memory complexes which are present in these tubes, represent subset of items that their weight are exceeded the capacity of knapsack. Then, the contents of tubes $T_{0}, T_{1}, T_{2}, \ldots, T_{c}$ are mixed together and transferred to tube $T_{0}$. Now, tube $T_{0}$ contains memory complexes which represent the subsets of items that their weight are not exceeded the capacity of knapsack. Furthermore, at the end of step 2, the memory complexes which represent the subsets of items that their weight are exceeded the capacity of knapsack, removed from solution space and only remain memory complexes representing subsets that fit in our knapsack. It is clear that the step 2 contains only 2 operations.

By the execution of step 3, sorting of memory complexes are performed according to the number of annealed stickers in $V$ region. During this step, $V+1$ tubes are produced $\left(T_{0}, T_{1}, T_{2}, \ldots, T_{V}\right)$. The memory complexes without any annealed stickers in $V$ region (represent the subset $\emptyset$ ) are placed in tube $T_{0}$, the memory complexes with only one annealed sticker (represent the subsets of items which their value are 1) are placed in tube $T_{1}$, the memory complexes with 2 annealed stickers (represent the subsets of items which their value are 2) are placed in tubes $T_{2}$, the memory complexes with 3 annealed stickers (represent the subsets of items which their value are 3 ) are placed in tube $T_{3}$, and finally, the tube $T_{V}$ contains the memory complexes with all bit regions located in $V$ region are turned to "on" (represent the subset which contains all items). Step 3 contains $V(V+1) / 2$ separate and $V(V+1) / 2$ combine operations, or totally it contains $V(V+1)$ operations. 
(1) For $i=n$ to $n+W-1$

For $j=i$ down to $n$

Separate $\left(T_{j-n}, i+1\right) \rightarrow\left(T_{(j-n+1)^{\prime}}, T_{j-n}\right)$

Combine $\left(T_{j-n+1}, T_{j-n+1}, T_{(j-n+1)^{\prime}}\right)$

(2) The capacity of knapsack is $C$,

Discard tubes $T_{c+1}, T_{c+2}, T_{c+3}, \ldots, T_{W}$

Combine $\left(T_{0}, T_{0}, T_{1}, T_{2}, \ldots, T_{c}\right)$

(3) For $i=n+W$ to $n+W+V-1$

For $j=i$ down to $n+W$

Separate $\left(T_{j-n-W}, i+1\right) \rightarrow\left(T_{(j-n-W+1)^{\prime}}, T_{j-n-W}\right)$

Combine $\left(T_{j-n-W+1}, T_{j-n-W+1}, T_{(j-n-W+1)^{\prime}}\right)$

(4) Read $T_{V}$; else if it was empty then:

Read $T_{V-1}$; else if it was empty then:

Read $T_{V-2}$; else if it was empty then:

:

Read $T_{2}$; else if it was empty then:

$\operatorname{Read} T_{1}$;

Algorithm 1

In step 4 , all of tubes (from $T_{V}$ to $T_{1}$ ) are evaluated for presence of memory complexes, and the first tube which is not empty and contains memory complexes represent the most valuable set. Step 4, maximally contains $V$ Read operations.

Finally, it is clear that the total number of operations in our algorithm is: $W^{2}+V^{2}+W+2 * V+2$.

\section{Conclusion}

In this paper, the sticker based DNA computing was used for solving the $0 / 1$ knapsack problem. This method could be used for solving other NP-complete problems. There are four principal operations in sticker model: Combination, Separation, Setting and Clearing. We also defined a new operation called "divide" and applied it in construction of solution space.

As mentioned earlier, one of the important properties of DNA computing is its real massive parallelism, which makes it a favorite and powerful tool for solving NP-complete and hard combinatorial problems. In sticker model, as in other DNA based computation methods, the property of DNA molecules to making duplexes is used as main biological operation. The main difference between the sticker model and Adleman-Lipton model is that in the sticker model there is a kind of Random access memory and the computations do not depend on DNA molecules extension as seen in AdlemanLipton model.

\section{References}

[1] L. Adleman, "Molecular computation of solutions to combinatorial problems," Science, vol. 266, pp. 1021-1024, 1994.

[2] S. Roweis, E. Winfree, R. Burgoyne et al., "A sticker based model for DNA computation," in Proceedings of the 2nd Annual Workshop on DNA Computing, Princeton University, L. Landweber and E. Baum, Eds., Series in Discrete Mathematics and Theoretical Computer Science, DIMACS, pp. 1-29, American Mathematical Society, 1999.

[3] R. J. Lipton, "DNA solution of hard computational problems," Science, vol. 268, pp. 542-545, 1995.

[4] L. M. Adleman, On Constructing a Molecular Computer, Department of Computer Science, University of Southern California, 1995.

[5] L. M. Adleman, “On constructing a molecular computer," in DNA Based Computers, R. J. Lipton and E. B. Baum, Eds., pp. 1-22, American Mathematical Society, 1996.

[6] W.-L. Chang and M. Guo, "Solving the dominating-set problem in Adleman-Liptons Model," in Proceedings of the 3rd International Conference on Parallel and Distributed Computing, Applications and Technologies, pp. 167-172, Kanazawa, Japan, 2002.

[7] W.-L. Chang and M. Guo, "Solving the clique problem and the vertex cover problem in Adleman-Lipton's model," in IASTED International Conference, Networks, Parallel and Distributed Processing, and Applications, pp. 431-436, Tsukuba, Japan, 2002.

[8] W.-L. Chang and M. Guo, "Solving NP-complete problem in the Adleman-Lipton Model," in Proceedings of The International Conference on Computer and Information Technology, pp. 157$162,2002$. 
[9] L. Adleman, P. Rothemund, S. Roweis, and E. Winfree, "On applying molecular computation to the data encryption standard," in Proceedings of the 2nd DIMACS wWorkshop on DNA Based Computers, Princeton University, pp. 24-48, 1996.

[10] H. Taghipour, A. Taghipour, M. Rezaei, and H. Esmaili, "Solving the independent set problem by sticker based DNA computers," American Journal of Molecular Biology, vol. 2, no. 2, pp. 153-158, 2012.

[11] Q. Ouyang, P. D. Kaplan, S. Liu, and A. Libchaber, "DNA solution of the maximal clique problem," Science, vol. 278, no. 5337, pp. 446-449, 1997.

[12] M. Amos, A. Gibbons, and D. Hodgson, "Error-resistant implementation of DNA computations," in Proceedings of the 2nd DIMACS Workshop on DNA Based Computers, 1996.

[13] M. Amos, A. Gibbons, and D. Hodgson, "A new model of DNA computation," in Proceedings of the 12th British Colloquium on Theoretical Computer Science, 1996.

[14] M. Hagiya, M. Arita, D. Kiga, K. Sakamoto, and S. Yokoyama, "Towards parallel evaluation and learning of boolean $\mu$ formulas with molecules," DIMACS Series in Discrete Mathematics and Theoretical Computer Science, vol. 48, pp. 57-72, 1999.

[15] E. Winfree, "Simulations of computing by self-assembly," in Proceedings of the 4th International Meeting on DNA Based Computers, pp. 213-239, 1998.

[16] E. Winfree, F. Liu, L. A. Wenzler, and N. C. Seeman, "Design and self-assembly of two-dimensional DNA crystals," Nature, vol. 394, no. 6693, pp. 539-544, 1998.

[17] E. Winfree, X. Yang, and N. Seeman, "Universal computation via self-assembly of DNA: some theory and experiments," in Proceedings of the 2nd DIMACS Workshop on DNA Based Computers, 1996.

[18] Q. Liu, Z. Guo, A. E. Condon, R. M. Corn, M. G. Lagally, and L. M. Smith, "A surface-based approach to DNA computation," in Proceedings of the 2nd Annual Meeting on DNA Based Computers, Princeton University, 1996.

[19] H. Taghipour, M. Rezaei, and H. Esmaili, "Applying surfacebased DNA computing for solving the dominating set problem," American Journal of Molecular Biology, vol. 2, no. 3, pp. 286-290, 2012.

[20] G. Rozenberg and H. Spaink, "DNA computing by blocking," Theoretical Computer Science, vol. 292, no. 3, pp. 653-665, 2003.

[21] M. R. Garey and D. S. Johnson, Computer and Intractability: a Guide to the Theory of NP-Completeness, Freeman, San Francisco, Calif, USA, 1979. 

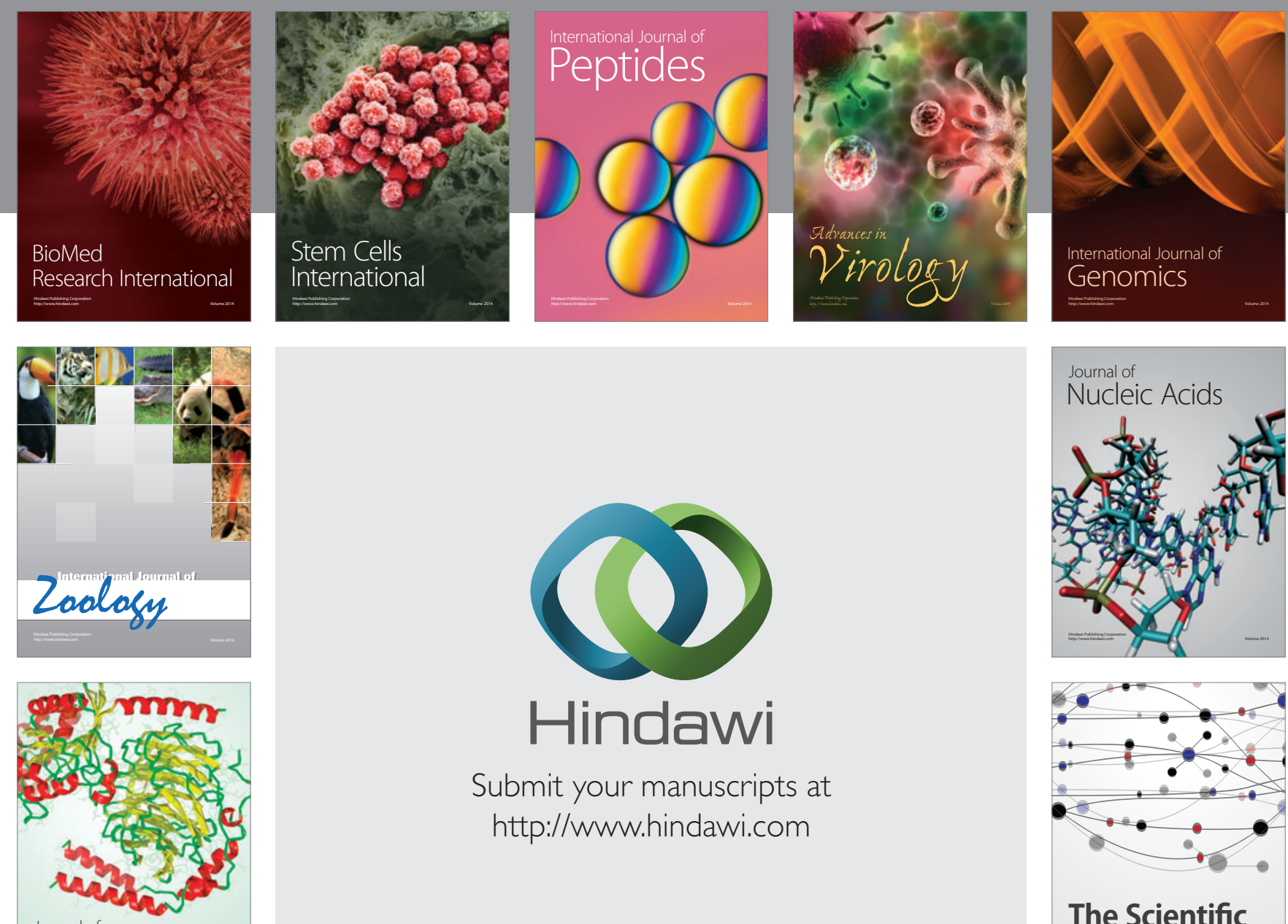

Submit your manuscripts at

http://www.hindawi.com

Journal of
Signal Transduction
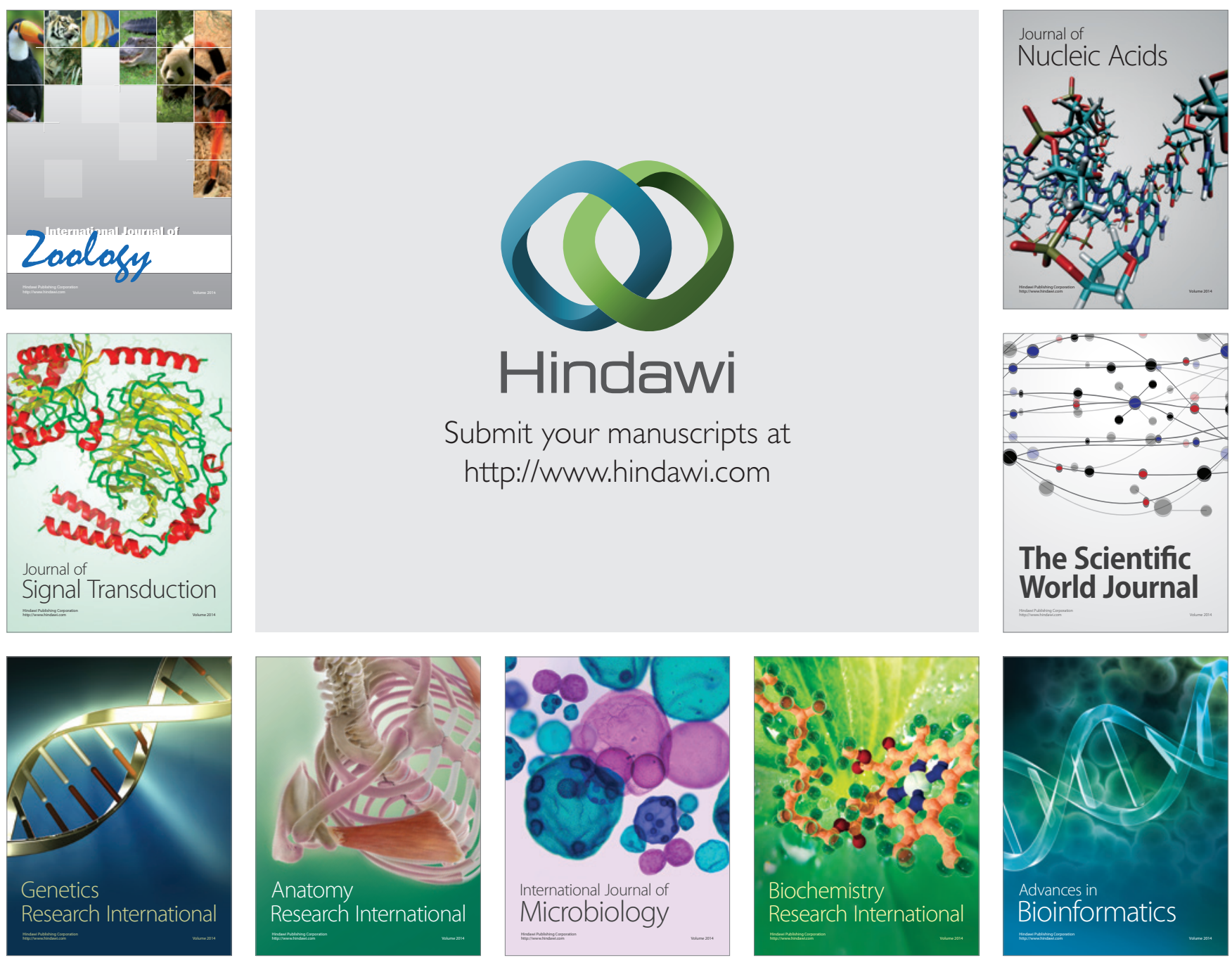

The Scientific World Journal
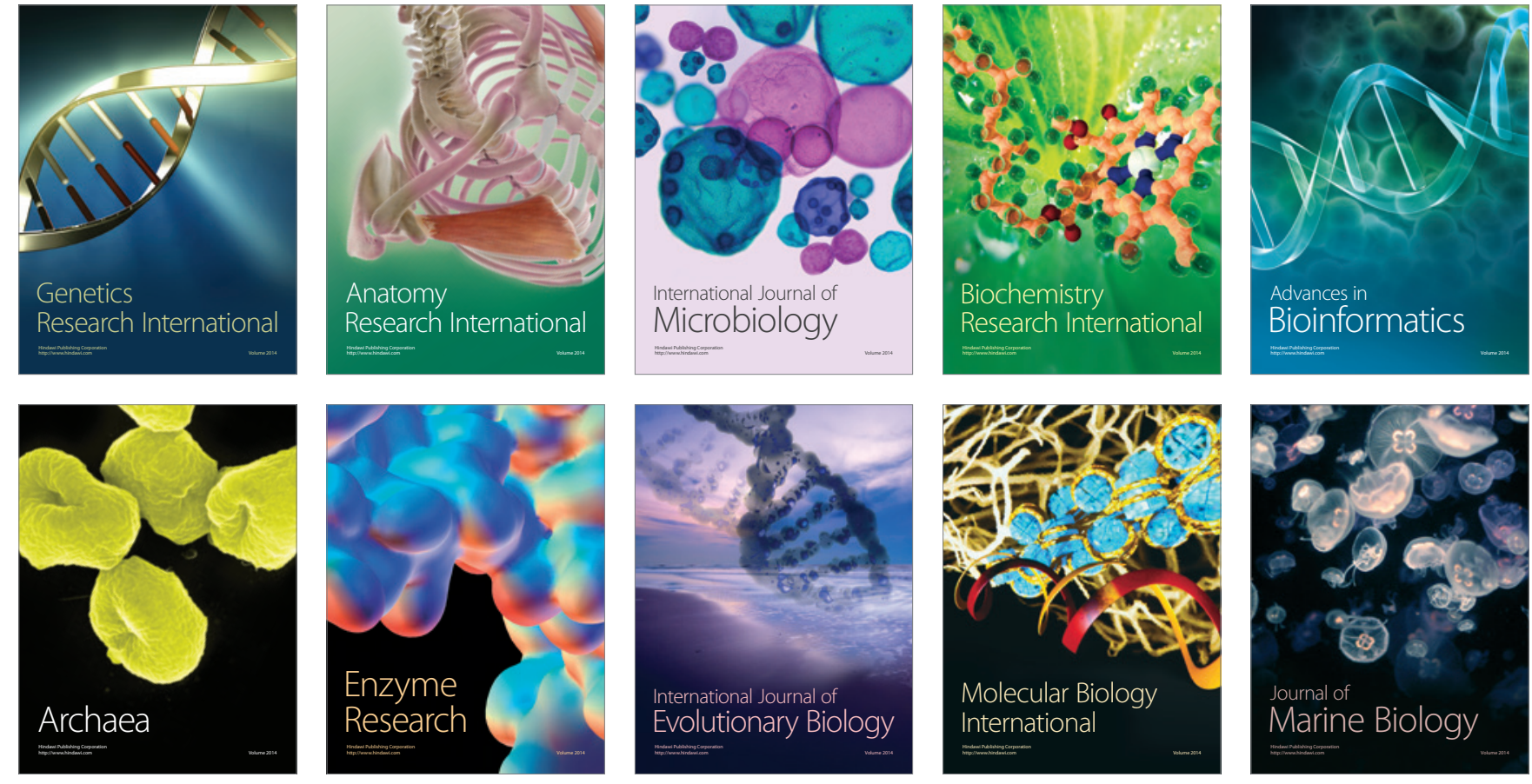